\title{
HEURISTIC EVALUATION CONDUCTED BY END USERS
}

\author{
Patrik Lif, Jonas Hermelin, Kristofer Bengtsson and Jonathan Svensson \\ FOI, The Swedish Defense Research Agency, 16490 Stockholm, Sweden
}

\begin{abstract}
Evaluation of interaction and user interface design is usually carried out by people specialized in usability and/or human factors. However, a common shortcoming for designers and evaluators is limited knowledge about how the systems are used in their context. Usability evaluation with heuristics is a common and rather fast method with which many important design issues and flaws can be discovered. However, it can be challenging to apply in domain-specific systems for non-domain experts. A study was therefore performed to evaluate how end users who are domain experts can be engaged to perform a heuristic evaluation on their main system of use. After being introduced to usability theory, design principles and the method of heuristic evaluation, the end users evaluated their system according to 17 selected usability heuristics. Their evaluation was part of a range of other usability assessments of the system. The results showed that the method is promising and the feedback from the end users was positive. It was also found that there is a need for additional training and more time for the evaluation. In future work, the evaluation method will be modified according to lessons learned and applied in other domain-specific systems.
\end{abstract}

\section{KEYWORDS}

Heuristic Evaluation, End Users, Interaction Design, Usability, Design Guidelines, Human-Computer Interaction

\section{INTRODUCTION}

Usability is a concept that encompasses a variety of qualities of products, systems and services, that together help users achieve their goals. Nielsen (1993a) described usability both as a method in the design process and as a quality attribute for assessing how easy interfaces are to use in five quality components: learnability, efficiency, memorability, errors, and satisfaction (Nielsen, 2012). There are several definitions of usability that highlight different aspects. One widely spread is described in the ISO standard 9241-11 (ISO, 1998): "The extent to which a product can be used by specified users to achieve specified goals with effectiveness, efficiency and satisfaction in a specified context of use." The purpose of the ISO 9241-11 standard is to focus on the outcome of product use in a specific context with certain goals, rather than describing usability as being a persistent property of a product (or system). The design must be adapted to an intended target group, situation, and the users' expected benefit of the product.

The ISO standard (ISO, 1998) offers a framework for usability, describing important aspects and information that should be included in the description and evaluation of usability, and their relationships. It is necessary to define the users, the product(s) or systems that are evaluated, the goals with the system or presentation, the context and situations in which it is used, and the tasks to be carried out to achieve the users' goals. The outcome of the interaction with the product is compared with the users' intended results of the interaction, which in turn relates to the users' goals. By measuring and evaluating aspects of effectiveness, efficiency and satisfaction, an indication of usability in a given context is provided.

Usability can be divided into the two main parts performance (broken down into effectiveness and efficiency) and satisfaction (Wichansky, 2017). The ISO standard (ISO, 1998) states that effectiveness, efficiency and satisfaction normally need to be measured separately with at least one measure for each component. The choice of measurement method should be adapted to the stated goal of the product or system, but the standard does not give any direct guidelines as to what dimensions should be chosen or how different measures should be combined, as the context may vary.

Different methods and evaluators provide different answers about where usability issues exist. System operators and usability experts may look at a system's usefulness, performance, effectiveness, efficiency and usability in different ways. The current paper concerns how usability can be evaluated by end users (system 
specialists but usability novices) who are introduced to heuristic evaluation, and what benefits and problems this method may entail. The procedure was performed in the context of other evaluation methods but this paper focuses on the heuristic evaluation.

\subsection{Heuristics for Interface Design and Evaluation}

The use of heuristics (i.e. principles or broad rules of thumb), to guide the design and evaluation of systems is widespread within the Human-Computer Interaction field. Normally, usability specialists perform a heuristic evaluation by applying a chosen set of proven design guidelines while interacting with the system or device, looking for issues where the design does not adhere to good practice or to the requirements of human perception and cognition. Found flaws can then be remedied early in the design process, avoiding major redesigns or unwanted interaction outcomes later on. Pierre (2015) listed a total of 33 sets of usability heuristics. The existing sets of heuristics are in many cases overlapping, but there are some differences due to diverse foci on specific contexts or types of system. Below are a selection of sets of design principles.

One prominent example of set of heuristics is Nielsen's (1993b, 2017) ten heuristics for user interface design and evaluation. The principles are: (1) visibility of system status, (2) match between system and the real world, (3) user control and freedom, (4) consistency and standards, (5) error prevention, (6) recognition rather than recall, (7) flexibility and efficiency of use, (8) aesthetic and minimalist design, (9) help users recognize, diagnose, and recover from errors, and (10) help and documentation (Nielsen, 1993b).

Norman (2013) has proposed six basic principles of interaction design that mainly focus on interaction with physical objects and how the artefact communicates with the users through its design. Norman's six principles are (1) consistency, (2) visibility, (3) affordance, (4) mapping, (5) feedback, and (6) constraints.

Shneiderman (2017) has put forth a list of eight "golden rules" for design that cover: (1) consistency, (2) shortcuts, (3) feedback, (4) dialogues with closure, (5) simple error handling, (6) easy reversal of actions, (7) internal control, and (8) reduced short-term memory load.

Tognazzini (2018) has given a comprehensive description of principles that are fundamental to the design of effective interfaces that should be both visually apparent and forgiving. In his work, Tognazzini has described a total of 75 principles divided into 19 areas: (1) esthetics, (2) anticipation, (3) autonomy, (4) color, (5) consistency, (6) defaults, (7) discoverability, (8) efficiency of the user, (9) explorable interfaces, (10) Fitt's law, (11) human interface objects, (12) latency reduction, (13) learnability, (14) metaphors, (15) protect users' work, (16) readability, (17) simplicity, (18) state, and (19) visible navigation.

Dix, Finlay, Abowd, and Beale (2004) have described a total of 14 principles in three main categories: learnability, flexibility and robustness. Learnability includes the five principles predictability, synthesizability, familiarity, generalizability, and consistency. Flexibility includes the five principles dialog initiative, multi-threading, task migratability, substitutivity, and customizability. Robustness includes the four principles observability, recoverability, responsiveness, and task conformance.

\subsection{Cognitive Walkthrough}

Cognitive walkthrough (Wharton et al., 1994) is a method for examining usability aspects by evaluating how participating end users carry out tasks using a given system. In contrast to heuristic evaluation, this is a task-specific approach to usability. The method takes it starting point in defining the task or tasks that users normally perform, which are then divided into simple processes that users follow. Questions during walkthrough entail user's goal, actions available, labelling and feedback. The questions differ both in number and content, depending on cognitive walkthrough variant (Blackmon et al., 2002; Mahatody et al., 2010).

\subsection{Usability Questionnaires}

User satisfaction is one of three key concepts within usability, and aspects of satisfaction can be measured using questionnaires. One example of a scientifically validated questionnaire is the System Usability Scale (SUS) (Bangor et al., 2008; Brooke 1996). All statements in SUS are to be answered on a five-point scale ranging from "Do not agree at all" to "Agree completely". The score ranges from 0-100. Bangor et al. (2008) collected a multitude of SUS results and recommended a scale for interpreting the score. A result exceeding 90 should be regarded as excellent, 80-90 as good, 70-79 as acceptable, 50-69 as doubtful with further 
improvement required, and scores below 50 should be considered as unacceptable. Even though SUS is a valuable instrument, it should not be used as the only measure of usability (Bangor et al., 2009) and therefore it needs to be supplemented with measurements of effectiveness and efficiency.

\section{CASE STUDY: EVALUATION OF A MARITIME SYSTEM}

As part of an evaluation of the usability of a maritime expert system, an adapted method was tested where end users conducted the heuristic evaluation. The system evaluation as a whole was based on the usability framework (ISO, 1998) as a starting point. Heuristic evaluation, cognitive walkthrough and questionnaires (SUS and a custom questionnaire) were used, but since this paper is on the use of heuristic evaluation with end users, only related results and experiences are presented. The end users were given an introduction and tools to evaluate the system in a structured and consistent manner. The clear advantage of this approach is that, unlike system developers and usability specialists, end users have a greater understanding of the actual use of the system and what effects a flaw may have. However, the end users in this paper did not have any prior experience of usability testing.

\subsection{Overall System Evaluation Process}

The work included task analysis, software review, selection of heuristics, system evaluation, and feedback to various organizations (Figure 1).

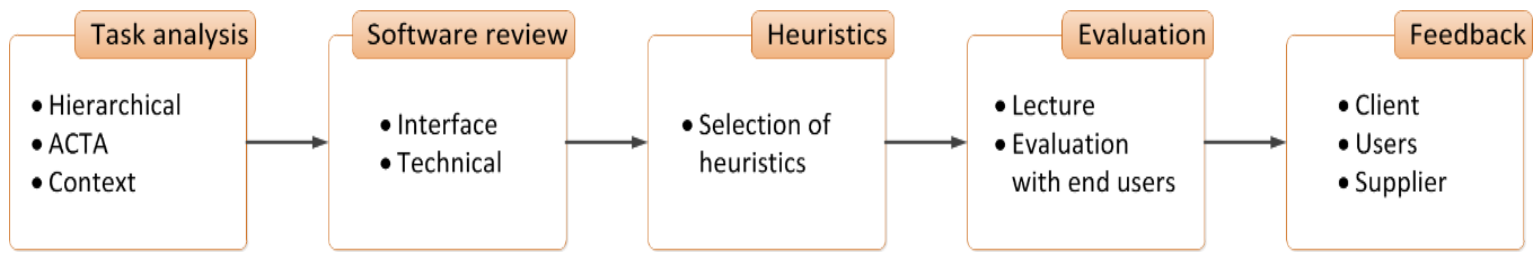

Figure 1. System evaluation process

To understand the context and environment in which the system is used, interviews were first conducted with the end users based on hierarchical task analysis (HTA) and applied cognitive task analysis (ACTA) (Stanton, Salmon, Walker, \& Jenkins, 2013). Complex challenging tasks were of particular interest since these imply special requirements for the system to function as intended. Visits were made to both the system supplier and the ships where the system was used, aimed to further understand the system and the context of use. In addition, discussions were held with end users about the strengths and weaknesses of the system.

Second, a software review was conducted where software documentation was studied. Third, a set of heuristics relevant for the system was selected. Fourth, a workshop was held where the system was evaluated through heuristic evaluation, cognitive walkthrough and questionnaires. Fifth, after the results were analyzed feedback was given to the client, users, and supplier.

\subsection{Selection of Heuristics}

Initially, a compilation of existing usability heuristics were gathered based on a literature review. From this compilation, the researchers selected a more narrow set of heuristics based on relevance to the system type, context and domain. The set of heuristics should (1) contain no duplicates, (2) cover perception, (3) error handling (4) expert systems, and (5) the number of heuristics should be limited. The perception category covered basic heuristics such as visibility, color, and readability. Some system limitations were known from the initial analysis, and therefore it was considered important to ensure good error prevention, error handling and help functions. As this was a system for users with different levels of experience, it was also important to include evaluation of automation, flexibility and shortcuts. The number of heuristics was limited to 17 (as well as the option Other, not displayed in Table 1) to ensure that the end users could learn all heuristics and to ensure they had time to analyze the system using them during the evaluation. A pamphlet of the heuristics was printed, containing icon, name, and a brief description of the heuristics (Table 1). 
Table 1. Heuristics selected for the evaluation. Icons by Flaticon (2019)

\begin{tabular}{|c|c|c|}
\hline$\overline{\text { Icon }}$ & Name & Description \\
\hline & Visibility & System status and description of what happens. \\
\hline \multirow{4}{*}{ (8) } & Color \& readability & Coloring and readability of text and symbols. \\
\hline & Delay visualization & Visualization of delays in the system so that users understand the status. \\
\hline & Autonomy & $\begin{array}{l}\text { Current system status. Ensure that users can take control of automatic } \\
\text { functions when needed. }\end{array}$ \\
\hline & Consistency & Use interfaces and word selection consistently. \\
\hline & $\begin{array}{l}\text { Match between system } \\
\& \text { real world }\end{array}$ & Use the users' language. System and reality compliance. \\
\hline & Users in control & $\begin{array}{l}\text { Support undo and redo. If users make a mistake, there should be support } \\
\text { to leave the status without redoing everything. }\end{array}$ \\
\hline & Flexibility & Support that users use the system differently. \\
\hline & Error prevention & Prevent users from committing errors. \\
\hline & Error handling & $\begin{array}{l}\text { Error messages should be expressed in a comprehensible manner. Specify } \\
\text { the problem and suggest solutions. }\end{array}$ \\
\hline & Help & $\begin{array}{l}\text { Use proactive help suggestions and clues. The help function should be } \\
\text { searchable and focus on tasks. Provide step-by-step instructions. }\end{array}$ \\
\hline \multirow[t]{2}{*}{ 洒: } & $\begin{array}{l}\text { Recognition rather } \\
\text { than recall }\end{array}$ & $\begin{array}{l}\text { Visible information, easy to perceive and recognize. Users should not } \\
\text { have to remember information. }\end{array}$ \\
\hline & Feedback & Feedback from the system so that actions are confirmed to the users. \\
\hline- & $\begin{array}{l}\text { Aesthetic \& } \\
\text { minimalism }\end{array}$ & $\begin{array}{l}\text { Use a minimalistic design and avoid information that is irrelevant or } \\
\text { rarely used. }\end{array}$ \\
\hline$\sum_{0}^{x}$ & Shortcuts & Shortcuts for frequently used commands. \\
\hline 目 & Save or send & $\begin{array}{l}\text { Save or send information so that users can continue to work from previous } \\
\text { status. }\end{array}$ \\
\hline & Igs & em's default settings. May apply to part or entire system. \\
\hline
\end{tabular}

\subsection{Evaluation with End Users}

The evaluated system was a maritime onboard computer system operated by a single operator. The interfaces of the system consist of a main display, a touch-input display, keyboard and trackball. The system is operated by specially trained personnel whose main tasks are performed within the system.

Eleven end users (ten men and one woman) were provided by the client to participate in the evaluation. The end users had one to ten years' experience working with the system. The least experienced individuals had a good overall knowledge of the particular system and a partially good knowledge of the ship's combined activities in daily operations. The most experienced end users can be viewed as experts, not only with regard to operating the designated system, but also with regard to other systems and the ship operations. None of the participants had previous experience of conducting heuristic evaluations.

The heuristic evaluation was carried out as part of a five hour long workshop consisting of five separate sessions. The first session was a 90-minute lecture on usability in general and evaluation methods in particular. During the lecture all the heuristics were explained and exemplified. The three sessions that followed consisted of 60 minutes for heuristic evaluation, 60 minutes for cognitive walkthrough, and 60 minutes for two questionnaires. The participants were divided into three groups and took part in each session in different order. Finally, an open discussion lasting 30 minutes was conducted with all end users and researchers.

The heuristic evaluation session was carried out on a system simulator. This allowed the end users to use the system in a similar way as onboard the ship. Although there were some limitations with stimuli feedback from certain hardware (e.g. sensors) of the system, the end users deemed the similarity to the onboard system to be good enough for this type of evaluation. 
As the number of simulators was limited, some participants had to work with the system in pairs. However, the evaluation and documentation of usability flaws in the interface were made individually. Each participant had access to the pamphlet with the 17 heuristics, complemented with a short description (Table 1). They were asked to note each usability flaw they could find for each heuristic in pre-prepared notebooks containing specified pages for each heuristic. If they were unable to match an identified flaw with a suitable heuristic, they were asked to categorize it as Other. The notes and categorizations were then submitted to the researchers for later analysis.

\section{RESULTS}

The results presented in this paper include the number of identified usability flaws the end users found for the 17 heuristics, the researchers' analysis of these flaws, and the participants' opinions of the system's usability and the evaluation method.

\subsection{Identified Usability Flaws}

The usability flaws identified by the users of the system were analyzed as follows: First, the end users identified, described and categorized usability flaws according to the 17 heuristics or as other. Most of the end users' identified flaws ( $71 \%$ of a total of 58$)$ were attributed to visibility, color \& readability, error prevention, and other, while the remaining 14 heuristics (29\%) were used to a lesser degree.

Second, the same usability flaw descriptions were interpreted and re-categorized by three researchers with knowledge about usability and heuristic evaluation. The purpose of this re-categorization was to achieve an overall understanding of how well the end users' categorization matched that of the researchers (Table 2). It should be noted that the sum of the number of flaws differs between end users and researchers since some of the end users' descriptions were split into multiple entities if they contained more than one flaw. No statistical analyses were made since only eleven end users and three researchers participated in the evaluation.

An analysis was made to evaluate the overlap between the end users' and the researchers' categorizations of the flaws identified by the end users (Table 2). The overlap corresponds to the proportion of the participants' statements that were categorized in the same way by the researchers. Calculation of overlap was omitted for heuristics with two or fewer statements from the end users. Some categories overlapped while others showed a large discrepancy. The end users' statements in the visibility heuristic had low overlap (29\%), and statements were mainly re-categorized to the heuristic color \& readability. Error prevention was erroneously used $(20 \%$ overlap) and the statements were re-categorized into a number of other heuristics. The heuristic color \& readability ( $83 \%$ overlap) and help (100\% overlap) contained fewer discrepancies between the end users' and the researchers' categorizations.

Table 2. Usability flaw categorization in heuristics by end users and researchers

\begin{tabular}{llll}
\hline Heuristic & End users & Researchers & \% overlap \\
\hline Visibility & 7 & 12 & 29 \\
Color \& readability & 12 & 20 & 83 \\
Delay visualization & 1 & 0 & - \\
Autonomy & 0 & 0 & - \\
Consistency & 0 & 2 & - \\
Match between system \& real world & 0 & 6 & - \\
Users in control & 0 & 0 & - \\
Flexibility & 1 & 5 & - \\
Error prevention & 10 & 5 & 20 \\
Error handling & 1 & 1 & - \\
Help & 4 & 5 & 100 \\
Recognition rather than recall & 2 & 6 & - \\
Feedback & 0 & 0 & - \\
Aesthetic \& minimalism & 1 & 7 & - \\
Shortcuts & 2 & 4 & - \\
Save \& send & 0 & 0 & - \\
Default settings & 0 & 0 & - \\
Other & 9 & 4 & - \\
\hline
\end{tabular}




\subsection{Subjective Feedback from End Users}

Overall, the participants verbally expressed that the heuristic evaluation worked well but that improvements could be made. The introductory lecture was found to be informative and the practical evaluation rewarding. The printed pamphlet was particularly appreciated by some participants because they could keep it and continue making evaluations of the system (or other systems) onboard their ship. Some of the end users who also participated in the task analysis that was performed about two months prior to the heuristic evaluation, expressed that it was of great value to conduct the two activities in close connection.

There were also suggestions on how the method could be improved. The respondents suggested that more time should be allocated to conducting evaluations in the simulator since several of them felt that one hour was not sufficient. One contributing aspect for this might be the large number of heuristics.

The respondents also suggested that more time should be allocated to the lecture on usability. Some of them highlighted that they had difficulties performing the heuristic evaluation due to the fact that it was their first time conducting such an evaluation and were lacking in experience. This could possibly be resolved by allocating more time for the lecture, thus giving the respondents more information. Other possibilities to remedy these difficulties are, for example, the development of more refined explanations on each of the heuristics, which in combination with a more extensive briefing could make it easier for the respondents to understand and perform the tasks given to them. According to the respondents, it would have been helpful to use the actual system instead of the simulator, which was not possible at the time of evaluation.

The evaluation workshop was rated highly appreciated as a whole $(\mathrm{M}=5.8)$, and the lecture was particularly appreciated $(M=6.2)$. The heuristic evaluation received positive feedback, but the participants' subjective ratings were lower (M=4.6) than the overall and lecture ratings (Figure 2).

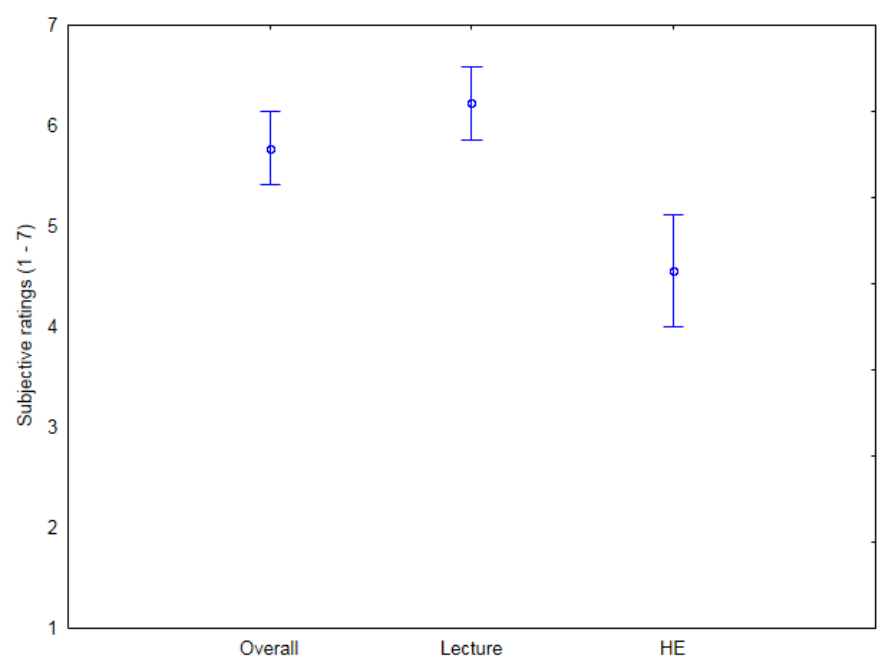

Figure 2. Mean and standard error of mean for subjective ratings of overall, lecture and heuristic evaluation (HE)

\section{DISCUSSION}

Heuristic evaluation is a well-known and recognized method for assessing the usability of systems, tools and interfaces. It is traditionally performed by designers or usability specialists as a formative evaluation in an iterative design process. Performing heuristic evaluations of specific complex systems in expert domains is difficult since extensive domain expertise is needed to comprehend all tasks and aspects of a system. A domain expert can on the other hand perform realistic tasks and thus identify critical flaws and their potential consequences. In the current study it was therefore evaluated if heuristic evaluation could be performed by end users who underwent a 90-minute introductory lecture on the basic principles of usability and how to perform a heuristic evaluation. 
The evaluation results are promising. The eleven end users felt that they were able to evaluate the system's usability to some degree (4.6 on a seven-grade scale) with heuristic evaluation, and they found a total of 58 usability problems or flaws. The researchers then re-coded these into 77 separate flaws into somewhat different categories. End users and usability specialists may complement each other by finding different flaws.

Most of the end users' identified flaws were mainly attributed to four heuristics (visibility, color \& readability, error prevention, and other). The cause for this distribution has not been investigated, but it is reasonable to assume that some of the heuristics are easier to understand and relate to in the evaluation of the system, while some categories of flaws are more rarely found in a deployed system. Usability flaws for the categories visibility and color \& readability are probably considerably easier to identify than autonomy and consistency related flaws. For example, it is easier to detect if text is too small or if windows are incorrectly designed, than how the interface is designed with regard to automation. Moreover, identifying usability flaws regarding system consistency can be more time consuming than identification of, e.g. readability flaws. The end users only had one hour to conduct the heuristic evaluation, which is likely to have limited the use of all the heuristics. For future studies, considerations should be made to reduce the number of heuristics and increase the time for the evaluation.

Although the lecture and heuristic evaluation were received positively, the end users reported some shortcomings in the method and process. The most obvious one was the short duration of both the lecture and the heuristic evaluation. In this study, a broad introduction to usability was chosen, including heuristic evaluation, cognitive walkthrough and SUS, as well as an evaluation using cognitive walkthrough, heuristic evaluation, SUS and a post-questionnaire. As the users only had seven hours at their disposal, the time for each activity became limited. Based on the feedback from the end users and according to the authors' own reflections, at least two hours are needed for the lecture and the heuristic evaluation, respectively. However, four hours or more would be adequate in order to give the users the opportunity to go through all relevant parts of the system. In this initial study, both the SUS questionnaire and a second overall questionnaire that together took one hour to answer were used. Future studies should probably focus more on one evaluation method, e.g. heuristic evaluation.

Some participants had problems classifying their identified flaws into the categories provided in the usability heuristics, which could be expected after only a 90-minute lecture. The conclusion is again that the lecture should be extended, and that it can be of value to focus more on the specific guidelines and somewhat less on usability overall during the introduction. However, when possible, an introduction should also include an overview of usability to give the end users a general understanding of the field and purpose, with the benefit of applying the knowledge on different systems they encounter in their work.

Perhaps the greatest benefit of having end users evaluate their own system is that there is no need to introduce them to the system. This is a big difference from when external designers or human factors experts evaluate a new system or design. Evaluating web sites or basic tools or systems is feasible, but evaluating a more advanced system in the context of other systems and a complex organization is considerably more challenging. This requires extensive training and several hours of use to understand the system and the context in which it is used. For some systems, several years of training may be required to become thoroughly knowledgeable. In these cases, it can be significantly more cost-effective to train end users to evaluate their own system under the direction of usability experts. Another option is to perform heuristic evaluation both with end users and usability experts and then cross-examine the outcomes to cover possible gaps in understanding and overcome the differences in perspective. The positive feedback from the participants who wished to keep the set of heuristics for future reference indicates that the heuristics could be useful as a way to guide how flaws in the system can be identified and reported during actual use.

In this initial study, end users' ability to apply heuristic evaluation by analyzing the usability flaws they identified was examined, along with their subjective experiences. In our continued work on methods of usability evaluation the intention is to make a comparison of results from heuristic evaluations conducted by end users, system designers, and usability specialists on an aviation system. The forthcoming study will expand the analysis and examine the differences between groups in more detail. It will also investigate if designers can identify usability flaws in the system they designed themselves.

With the suggested methodological modifications, heuristic evaluation performed by end users could be a favorable and cost-effective way to identify usability flaws in certain complex and domain-specific systems. 


\section{REFERENCES}

Bangor, A., Kortum, P., \& Miller, J. (2009). Determining What Individual SUS Scores Mean: Adding an Adjective Rating Scale. Journal of Usability Studies, 4(3), 114-123.

Bangor, A., Kortum, P. T., \& Miller, J. T. (2008). An Empirical Evaluation of the System Usability Scale. International Journal of Human-Computer Interaction, 24(March 2015), 574-594.

Blackmon, M., Polson, P., Kitajima, M., \& Lewis, C. (2002). Cognitive Walkthrough for the Web. I CHI 2002 (s. Vol. 4, No. 1. pp. 463-470). Minneapolis, Minnesota, USA: ACM.

Brooke, J. (1996). SUS -A quick and dirty usability scale Usability and context.

Brooke, J. (2013). SUS : A Retrospective. Journal of Usability Studies, 8(2), 29-40.

Dix, A., Finlay, J., Abowd, G. D., \& Beale, R. (2004). Human-Computer Interaction (Third Edition). Harlow, England: Pearson Prentice Hall.

Flaticon. (2019). Flaticon. Retrieved 01 februari 2019, from https://www.flaticon.com

ISO. (1998). ISO 9241-11:1998 - Ergonomic requirements for office work with visual display terminals (VDTs) -- Part 11: Guidance on usability. SIS. Stockholm.

Lewis, J. R., \& Sauro, J. (2009). The Factor Structure of the System Usability Scale (s. 94-103). Springer, Berlin, Heidelberg.

Mahatody, T., Sagar, M., \& Kolski, C. (2010). State of the Art on the Cognitive Walkthrough Method, Its Variants and Evolutions. International Journal of Human-Computer Interaction, 26(8), 741-785.

Nielsen, J. (1993a). Iterative user-interface design. Computer, 26(11), 32-41.

Nielsen, J. (1993b). Usability Engineering. San Diego: Academic Press.

Nielsen, J. (2012). Usability 101: Introduction to Usability. From https://www.nngroup.com/articles/usability-101introduction-to-usability/

Nielsen, J. (2017). 10 Usability Heuristics for User Interface Design. From https://www.nngroup.com/articles/ten-usabilityheuristics/

Norman, D. (2013). The Design of Everyday Things (2nd ed.). Basic books.

Pierre, R.S.D.S.D.Q. (2015). Heuristics in Design: A Literature Review. Procedia Manufacturing, 3, 6571-6578.

Shneiderman, B. (2017). The Eight Golden Rules of Interface Design. Hämtad 15 november 2017, from https://www.cs.umd.edu/users/ben/goldenrules.html

Stanton, N. A., Salmon, P. R., Walker, G. B., \& Jenkins, D. (2013). Human Factors Methods: A Practical Guide for Engineering and Design. Surrey, England: Ashgate Publishing Limited.

Tognazzini, B. (2018). First Principles of Interaction Design. From https://asktog.com/atc/principles-of-interaction-design/

Wharton, C., Rieman, J., Lewis, C., \& Polson, P. (1994). The cognitive walthrough Method: A Parctitioner's Guide. I J. Nielsen \& R. Mack (Red.), Usability Inspection Methods. New York.

Wichansky, A. (2017). Hands-On Software Usability Testing Lab and Advanced Methods. HCI International. 